\title{
ANÁLISIS IN VITRO E IN VIVO DE SOBRENADANTES CRUDOS DE Clostridium perfringens AISLADOS DE CASOS DE ENTEROTOXEMIA EN ALPACAS
}

\author{
IN VITRO AND IN VIVO ANALYSIS OF CLOSTRIDIUM PERFRINGENS SUPERNATANT \\ ISOLATES From AlPACA ENTEROTOXEMIA CASES
}

\author{
David Pérez J. ${ }^{1,4}$, Luis Llanco A. ${ }^{1}$, Hugo Castillo D. ${ }^{2}$, Álvaro Véliz A. ${ }^{2}$, Iván \\ Carhuallanqui P. ${ }^{1}$, Luis Luna E. ${ }^{1}$, Pablo Londoñe B. ${ }^{1}$, Roxane Piazza F. ${ }^{3}$, \\ Raúl Rosadio A. ${ }^{1,2}$, Lenin Maturrano H. ${ }^{1}$
}

\section{RESUMEN}

Se reportan evaluaciones de sobrenadantes bacterianos (nativos) crudos obtenidos de 24 aislados de $C$. perfringens de casos fatales de enterotoxemia buscando caracterizar propiedades lecitinasas, hemolíticas, citotóxicas in vitro y enterotóxicas in vivo de la exotoxina fosfolipasa C (Cp-PLC). Los aislados (12 en estados vegetativos y 12 esporulados) contenían el gen cpa (genotipo A), y seis de ellos tenían además el gen $c p b 2$, pero todos carecían del gen de la enterotoxina (cpe). Las actividades lecitinasas fueron evaluadas, en microplacas, usando emulsiones de yemas de huevo; las hemolíticas y perfringolisina en microplacas, usando eritrocitos de carnero y de equino respectivamente; las citotóxicas, en células HEp-2; y las enterotóxicas en conejos inoculados intraintestinalmente. Los aislados fueron clasificados, de acuerdo a los niveles de la actividad lecitinasa, en cepas de alta, mediana y baja producción de Cp-PLC. La mayoría de aislados $(66.6 \%)$ en estados vegetativos mostraron perfiles de alta y mediana producción de Cp-PLC, mientras que la totalidad de los esporulados fueron de baja producción. Todos los aislados altamente productores de Cp-PLC fueron citotóxicos y mostraron las mayores actividades hemolíticas $(24.0 \pm 8.4$ u.a.h). A pesar de que el 66.6\% $(n=16)$ de los aislados indujeron acumulación de fluido intestinal $(0.12-0.47 \mathrm{~mL} / \mathrm{cm})$, ninguno fue capaz de generar lesiones intestinales en conejos, similares a las descritas en la enterotoxemia. Los sobrenadantes crudos de los aislados evidenciaron la presencia de otras toxinas tipo hemolisinas, citotoxinas y enterotoxinas, distintas a Cp-PLC, CPE y toxina $\hat{a} 2$, que necesitan ser investigadas a fin de elucidar su posible rol en la etiopatogénesis de la enterotoxemia en alpacas.

Palabras clave: Clostridium perfringens, enterotoxemia, Cp-PLC

\footnotetext{
${ }^{1}$ Laboratorio de Microbiología y Parasitología Veterinaria, Facultad de Medicina Veterinaria, Universidad Nacional Mayor de San Marcos, Lima, Perú

${ }^{2}$ CONOPA - Instituto de Investigación y Desarrollo de Camélidos Sudamericanos, Lima, Perú

${ }^{3}$ Laboratorio de Bacteriología, Instituto Butantan, São Paulo, Brasil

${ }^{4}$ E-mail:dapeja84@gmail.com
} 
In the present study, 24 native supernatants isolated from enterotoxemia fatalities were evaluated to characterize lecitinase, hemolytic, in vitro cytotoxicity, and in vivo enterotoxicity activities of the phospholipase $\mathrm{C}$ (Cp-PLC) exotoxin. The lecitinase activity was monitored in microplates using egg white emulsifications, the hemolityc and perfringolisine in microplates using sheep and equine eritrocytes respectively, the cytotoxic activity was tested on HEp-2 cells and the enterotoxicity by inoculating within intestinal loops of rabbits. At the molecular level, all the isolates (12 vegetative and 12 sporulated) were found to contain the cpa gene (genotype A), while six also contained cpb2 gene but none had the cpe gene. These isolates were classified, according the lecitinase activity, as high, medium and low producer strains. Most of the vegetative $(66.6 \%)$ isolates had high and medium Cp-PLC production profiles, whereas all of the sprorulated ones were low producers. All high lecitinase production strains were citotoxic to $\mathrm{HeP} 2$ cells and the highest hemolytic activities (24.0 \pm 8.4 u.a.h). Although the $66.6 \%$ $(\mathrm{n}=16)$ of the isolates induced intestinal fluid accumulations $(0.12-0.47 \mathrm{~mL} / \mathrm{cm})$ none of the 24 isolates was able to produce intestinal lesions similar to enterotoxemia. The native supernantants showed presence of other toxins with hemolytic, citotoxic and enterotoxin activities similar to the Cp-PLC, CPE and $\hat{a} 2$ toxin that warrant further investigation to elucidate their possible role in the etiopathogenecity of enterotoxemia in alpacas.

Key words: Clostridium perfringens, enterotoxemia, Cp-PLC

\section{INTRODUCCIÓN}

La enterotoxemia es la enfermedad más devastadora que afecta a la población de alpacas en el Perú, provocando hasta 70\% de mortalidad en las crías (Ramírez, 1991). Las lesiones son de tipo necrótico y hemorrágica, afectando principalmente el yeyuno e íleon, que derivan en una toxemia, ocasionando muerte súbita en la mayoría de los casos (Moro, 1987; Ameghino y DeMartini, 1991). Recientes análisis moleculares realizados en 47 aislados de $C$. perfringens corroboran que la mayor parte (98\%) pertenece al genotipo A, sugiriendo a la Cp-PLC (C. perfringens PhosphoLipase C), y en algunos casos, a la toxina $\hat{a} 2$ como los principales factores toxigénicos $\mathrm{y}$, asimismo, descartando la participación de la CPE (C. perfringens Enterotoxin) en la patogénesis de la enterotoxemia en alpacas (Pérez, 2006).
La Cp-PLC es una zinc-metalofosfolipasa con marcada preferencia por dos principales componentes de la capa externa de las membranas celulares, la fosfatidilcolina y la esfingomielina (Nagahama et al., 1996). In vivo, la Cp-PLC es potencialmente letal pues incrementa la contractibilidad cardiaca, aumenta la permeabilidad capilar, induce agregación plaquetaria, hemolisis y mionecrosis (Titball et al., 1999). Las actividades de CpPLC son dependientes de su capacidad para hidrolizar membranas fosfolipídicas, donde en bajas concentraciones genera segundos mensajeros, como el diacilglicerol y la ceramida, que gatillan señales de transducción que conducen a la producción descontrolada de mediadores intercelulares, mientras que en altas concentraciones causa una masiva degradación fosfolipídica, disrupción de membrana y citolisis (Titball et al., 1999).

La Cp-PLC ha demostrado ser un factor de virulencia esencial e imprescindible para la patogénesis de la gangrena gaseosa (Flores-Díaz y Alape-Girón, 2003; Titball, 2005), 
y además se le postula como factor de virulencia clave en la patogénesis de muchas enteropatías causadas por $C$. perfringens tipo A carente de genes cpe y cpb2 [cpe${ }^{\mathrm{v}} c p b 2^{\text {-vo }}$ (no enterotoxigénico ni $\hat{a} 2$ toxigénico)] tales como la enteritis necrótica en pollos y en lechones (Gholamiandekhordi et al., 2006; Kanakaraj et al., 1998), disentería hemorrágica en corderos (Gkiourtzidis et al., 2001), y enterotoxemia en corderos, venados y alpacas (Moro, 1987; Embury-Hyatt et al., 2005; Kalender et al., 2005; Pérez, 2006). Sin embargo, existe muy poca información que demuestre su directa participación en los cuadros entéricos.

En el presente estudio se evaluó 24 sobrenadantes bacterianos crudos de aislados de C. perfringens obtenidos de casos de enterotoxemia en alpacas buscando determinar la producción de la Cp-PLC, mediante su actividad lecitinasa, y ciertas características toxigénicas tales como la actividad hemolítica, citotóxica y, sobre todo, capacidad enterotóxica en conejos.

\section{Materiales y MéTodos}

\section{C. perfringens Aislados de Casos de Enterotoxemia en Alpacas}

Se emplearon 24 aislados de $C$. perfringens obtenidos de casos de enterotoxemia en alpacas, 12 en estados vegetativos y 12 esporulados. Todos contenían el gen cpa (genotipo A) pero negativos al gen cpe codificante de la CPE. Seis de estos 24 aislados contenían, además, el gen cpb2 (subtipo $\hat{a} 2^{+ \text {vo }}$ ) (Cuadro 1 ).

\section{Cultivos Bacterianos y Obtención del Sobrenadante}

Los aislamientos bacterianos, adecuadamente genotipificados, fueron inoculados en caldo Breath Heart Infusion (1\%, v/v) e incubados bajo condiciones de anaerobiosis a $37^{\circ} \mathrm{C}$ durante $17 \mathrm{~h}$, tiempo necesario para obtener una mayor síntesis de Cp-PLC (Pérez, 2010). Posteriormente, los cultivos fueron centrifugados a $13000 \mathrm{x} g$ durante 10 min a $4{ }^{\circ} \mathrm{C}$ para recuperar sobrenadantes y adicionarles inhibidores de proteasas (fluoruro de fenilmetilsulfonilo) a una concentración final de $1 \mathrm{mM}$ y ser conservados, hasta su uso, a $-20^{\circ} \mathrm{C}$.

\section{Capacidad de Producción de Cp-PLC por C. perfringens}

Los niveles de producción de Cp-PLC de los aislados fueron determinados observando actividades lecitinasas. Se utilizaron microplacas de 96 pocillos, depositando 100 ì L de sobrenadante bacteriano mezclados con 100 ì L de emulsión de yema de huevo (10\% [ $\mathrm{vol} / \mathrm{vol}]$ de yema de huevo en PBS $1 \mathrm{X}[\mathrm{pH}$ 7.3]) e incubando a $37{ }^{\circ} \mathrm{C}$ durante $3 \mathrm{~h}$. La actividad enzimática fue determinada utilizando un lector de ELISA con un filtro de 620 $\mathrm{nm}\left(\mathrm{OD}_{620 \mathrm{~mm}}\right)$ de densidad óptica, teniendo como muestra blanco un pozo con 100 ì L de BHI estéril mezclado con 100 ì L de emulsión yema de huevo. La actividad lecitinasa fue expresada en unidades arbitrarias (u.a.) y fue el resultado después de dividir la diferencia de los valores de $\mathrm{OD}_{620 \mathrm{~nm}}$ de la muestra y el $\mathrm{OD}_{620 \mathrm{~nm}}$ del blanco entre el valor de $\mathrm{OD}_{620 \mathrm{~nm}}$ del blanco. Los aislados con valores inferiores a 1 u.a., de 1-3 u.a, y superiores a 3 u.a. fueron clasificados como aislados de baja, mediana y alta capacidad de producción de Cp-PLC.

\section{Caracterización Toxigénica de los $C$. perfringens Aislados}

\section{Actividad hemolitica}

Se determinó siguiendo la metodología descrita por Titball et al. (1989). Brevemente, el sobrenadante de cada aislado fue diluido en forma seriada $\left(1 / 2\right.$ hasta $\left.1 / 1024 ;\left[\log _{2}\right]\right)$ en buffer borato isotónico (BBI; $0.57 \mathrm{~g}$ de $\mathrm{NaB}_{4} \mathrm{O}_{7} \cdot 10 \mathrm{H}_{2} \mathrm{O}, 2.1 \mathrm{~g}$ de $\mathrm{H}_{3} \mathrm{BO}_{3}, 1.8 \mathrm{~g}$ de $\mathrm{CaCl}_{2} \cdot 2 \mathrm{H}_{2} \mathrm{O}$, y $7.5 \mathrm{~g}$ de $\mathrm{NaCl}$ disueltos en $1 \mathrm{~L}$ de agua destilada [pH 7.6]) en una microplaca de 96 pocillos hasta alcanzar volú- 
menes finales de 150 ì L. Se agregó a cada pocillo 150 ì L de la solución de eritrocitos de carnero (resuspendidos en BBI al 1\% [vol/ vol]). La microplaca fue incubada durante 1 h a $37{ }^{\circ} \mathrm{C}$ seguida de otra hora a $4{ }^{\circ} \mathrm{C}$, buscando evidenciar la hemólisis calor-frío característica de la Cp-PLC. La microplaca fue posteriormente centrifugada a $2000 \mathrm{x} g$ durante 10 min a $4{ }^{\circ} \mathrm{C}$ para obtener 200 ì $\mathrm{L}$ de cada sobrenadante, y ser transferida a otra microplaca para ser leída en el lector ELISA con filtro de densidad óptica de $550 \mathrm{~nm}$ $\left(\mathrm{OD}_{550 \mathrm{~nm}}\right)$. La actividad hemolítica fue expresada como unidades arbitrarias hemolíticas (u.a.h.) por mililitro que correspondieron a la dilución en la cual se observa $50 \%$ de hemólisis. La prueba tuvo como referencia la $\mathrm{OD}_{550 \mathrm{~nm}}$ de una dilución de la solución de eritrocitos en agua destilada (vol/vol, 1:3), que correspondió a una hemólisis del 50\%.

\section{Actividad perfringolisina}

Esta actividad permitió evaluar la expresión específica de otra hemolisina de $C$. perfringens conocida como la perfringolisina (PFO). Se determinó siguiendo la metodología descrita por Fisher et al. (2006), para la cual el sobrenadante de cada aislado fue seriadamente diluida $\left(1 / 2\right.$ hasta $\left.1 / 128 ;\left[\log _{2}\right]\right)$ en BBI suplementado con $1 \mathrm{mM}$ EDTA (inhibidor de la Cp-PLC) hasta alcanzar volúmenes finales de 150 ì L y ser distribuidas en una microplaca de 96 pocillos. Se agregó a cada pocillo 150 ì L de solución de eritrocitos de caballo [resuspendidos en BBI suplementado con $1 \mathrm{mM}$ EDTA al 1\% (vol/vol)]. La placa fue incubada a $37^{\circ} \mathrm{C}$ durante $1 \mathrm{~h}$ y centrifugada a $2000 \mathrm{x} g$ durante $10 \mathrm{~min}$ a $4{ }^{\circ} \mathrm{C}$ para obtener $200 \mathrm{ì} \mathrm{L} \mathrm{de} \mathrm{cada} \mathrm{sobrenadante} \mathrm{y}$ ser transferidas a otra microplaca y leída en el lector de ELISA con filtro de densidades de $\mathrm{OD}_{550 \mathrm{~mm}}$. La actividad hemolítica fue expresada como unidades arbitrarias hemolíticas (u.a.h.) por mililitro que correspondieron a la dilución en la cual resultó el 50\% de hemolisis. La prueba tuvo como referencia la lectura de $\mathrm{OD}_{550 \mathrm{~mm}}$ de una dilución de la solución de eritrocitos en agua destilada (vol/vol, 1:3), que correspondió a una hemólisis del 50\%.

\section{Actividad citotóxica}

Los efectos citotóxicos fueron evaluados en una línea celular derivada de carcinoma epidermoide de laringe humano (células HEp-2), utilizando una placa de 24 pocillos conteniendo en el fondo laminillas adheridas con monocapas celulares en grado de semiconfluencia y 800 ì L de Medio Dulbecco's Modified Eagle's (DMEM) suplementado con $2 \%$ de suero fetal bovino y $1 \%$ de manosa. A esta placa se agregó, suavemente, 200 ì L de cada sobrenadante bacteriano y fue incubada inmediatamente, a $37^{\circ} \mathrm{C}$ durante $24 \mathrm{~h}$ en estufa de $\mathrm{CO}_{2}$. Después de la incubación, las laminillas fueron retiradas para ser fijadas en metanol 70\%, coloreadas con Giemsa y depositadas en láminas portaobjetos. Los efectos citotóxicos fueron determinados mediante evaluación microscópica a 40X analizando morfología o alteración celular.

\section{Actividad enterotóxica}

Se evaluó en tres conejos de raza Nueva Zelanda de aproximadamente $3 \mathrm{~kg}$ de peso, adaptando la prueba de asa intestinal ligada, descrita por Duncan et al. (1968), Fiore et al. (1997) y Campos et al. (2004). En resumen, se practicó una anestesia profunda al animal con clorhidrato de ketamina (30 mg/ $\mathrm{kg}$ pv, vía im) asociada a clorhidrato de xilacina ( $3 \mathrm{mg} / \mathrm{kg}$ pv, vía i.m.), y se realizó una incisión en la línea media ventral a nivel de la cicatriz umbilical para exponer y ligar segmentos de íleon de aproximadamente $5 \mathrm{~cm}$. Se generaron un total de ocho asas intestinales ligadas (AIL) por conejo incluyendo un control en cada individuo. Cada AIL fue intraluminalmente inoculada, en forma aséptica, con $500 \mu \mathrm{L}$ de sobrenadante bacteriano de cada aislado, y el AIL control inoculado con $500 \mu \mathrm{L}$ de medio BHI. Los animales fueron mantenidos en anestesia general y analgesia profunda (Tramadol $[10 \mathrm{mg} / \mathrm{kg} \mathrm{pv}$, vía im]) durante todo el ensayo (24 horas). Pasado el periodo de observación, los animales fueron sacrificados con sobredosis de pentobarbital sódico $(200 \mathrm{mg} / \mathrm{kg}$ pv, vía endovenosa) y se procedió a la evaluación 
observando capacidad de acumulación de fluido intestinal mediante la relación volumen $(\mathrm{mL})$ de fluido intestinal por longitud $(\mathrm{mm})$ del AIL, y los cambios histopatológicos a nivel de mucosa, submucosa y serosa intestinal en cortes de tejidos fijados y coloreados con Hematoxilina y Eosina.

\section{Resultados y Discusión}

El análisis evidencia, principalmente en estados vegetativos, la presencia de Cp-PLC en su forma nativa mostrando actividades de lecitinasa con tres diferentes niveles de producción (altos, medianos y bajos) que contrasta con 11/12 aislados esporulados que fueron de baja producción (Cuadro 1).

El 41.6\% (5/12) de los aislados en estado vegetativo fueron altamente productores, $25 \%(3 / 12)$ de mediana producción y el restante $33 \%$ (4/12) de baja producción de CpPLC. Por otro lado, 11 de los 12 aislados en estados esporulados fueron clasificados dentro del perfil de baja producción de Cp-PLC (Cuadro 1). Estos resultados corroboran que la Cp-PLC se sintetiza principalmente durante la fase logarítmica de crecimiento bacteriano (Ba-Thein et al., 1996).

Las variaciones en los niveles de las actividades de lecitinasa entre los aislados de C. perfringens podrían ser consecuencia de la presencia de promotores reguladores o mutaciones en regiones extragénicas y no como producto de mutaciones en la región codificante, pues el gen cpa de los cinco (AE) tipos de $C$. perfringens aislados de animales sanos y enfermos y de cepas altas y bajas productoras de Cp-PLC son altamente homólogos y similares (Katayama et al., 1993; Tsutsui et al., 1995; Sheedy et al., 2004). La presencia de tres perfiles de producción de Cp-PLC en aislados de casos de enterotoxemia en alpacas sugiere la presencia de $C$. perfringens con varios grados de virulencia o patogenicidad en los animales infectados. Similares resultados han sido re- portados en otras dolencias causadas por $C$. perfringens (Tsutsui et al., 1995; Bullifent et al., 1996; Timbermont et al., 2009).

Por otra parte, el análisis de los seis aislados genotipificados como subtipo $\hat{a} 2^{+\mathrm{vo}}(2$ vegetativos y 4 esporulados), cuya toxina es un factor de virulencia implicada en enterotoxemia de terneros (Manteca et al., 2002) y enteritis necrótica en cerdos (Schotte et al., 2004), demostró que el 83.3\% (5/6) tenía un perfil de baja producción de Cp-PLC (Cuadro 2). La baja capacidad productora de $\mathrm{Cp}$ PLC en la mayoría de estos aislados es intrigante pues este subtipo fue detectado en el $13.7 \%$ de los aislados de casos de enterotoxemia de alpacas que se llegó a sospechar de una posible interacción con la $\mathrm{Cp}$ PLC para desencadenar una forma más severa de enterotoxemia (Pérez, 2006). Sin embargo, no puede descartarse el posible rol en la patogénesis de la enfermedad pues el estudio evaluó, aparentemente, solamente la toxina codificada por el gen cpa.

Los aislados en estados vegetativos mostraron la mayor actividad hemolítica (26.3 \pm 29.1 u.a.h. $/ \mathrm{mL}$ ) comparado con 1 os esporulados (1.2 \pm 1.64 u.a.h. $/ \mathrm{mL})$ (Cuadro 3). La actividad hemolítica no guardó una relación proporcional con la capacidad de producción de Cp-PLC, sino contrariamente, los aislados en estado vegetativo bajos productores fueron los más hemolitícos (49.5 \pm 59.7 u.a.h./mL), quedando los aislados de mayor producción con menores capacidades hemolíticas ( $24 \pm 8.4$ u.a.h./mL) (Cuadro 3). Estas aparentes contradicciones sugieren la(s) presencia(s) y/o producción(es) de otra(s) hemolisina(s) distintas pero de similares características a Cp-PLC, que tal vez expliquen lo observado en un aislado de baja producción de Cp-PLC que mostró la mayor actividad hemolítica $(96.0 \pm 45.3$ u.a.h./mL). Este resultado evidencia, además, la posible existencia de aislados con distintas capacidades patogénicas, no necesariamente relacionados con actividades lecitinasas. Ninguno de los aislados subtipo $\hat{a} 2^{+\mathrm{vo}}$ mostró actividad hemolítica, lo cual podría explicarse por 
Cuadro 1. Capacidad de producción de Cp-PLC y niveles de actividades hemolíticas, perfringolisinas, citotóxicas y acumulación de fluido intestinal de aislados de $C$. perfringens de casos de enterotoxemia en alpacas

\begin{tabular}{|c|c|c|c|c|c|c|c|c|}
\hline $\mathrm{N}^{\circ}$ & $\begin{array}{c}\text { Estado } \\
\text { bacteriano }\end{array}$ & Genotipo $^{2}$ & $\begin{array}{l}\text { Actividad } \\
{ }^{2} \text { lecitinasa }{ }^{3} \\
\text { (u. a. } / \mathrm{mL} \text { ) }\end{array}$ & $\begin{array}{l}\text { Capacidad } \\
\text { de } \\
\text { producción } \\
\text { de Cp- } \\
\text { PLC }^{4}\end{array}$ & $\begin{array}{l}\text { Actividad } \\
\text { hemolítica } \\
\text { (u.a.h. } / \mathrm{mL} \text { ) }\end{array}$ & $\begin{array}{c}\text { Actividad } \\
\text { perfringolisina } \\
\text { (u.a.h. } / \mathrm{mL})\end{array}$ & $\begin{array}{l}\text { Actividad } \\
\text { citotóxica }^{7}\end{array}$ & $\begin{array}{l}\text { Acumulación } \\
\text { de fluido } \\
\text { intestinal }^{8} \\
(\mathrm{~mL} / \mathrm{cm})\end{array}$ \\
\hline 1 & Vegetativo & $\mathrm{A} \beta 2^{+v o s}$ & $-0.2 \pm 0.01$ & Baja & $0 \pm 0.0$ & $0 \pm 0$ & - & 0.00 \\
\hline 2 & Vegetativo & A $\beta 2^{+v o s}$ & $-0.2 \pm 0.01$ & Baja & $0 \pm 0.0$ & $0 \pm 0$ & + & 0.00 \\
\hline 3 & Vegetativo & A & $0.8 \pm 0.05$ & Baja & $3 \pm 1.4$ & $2 \pm 0$ & - & 0.21 \\
\hline 4 & Vegetativo & A & $0.0 \pm 0.04$ & Baja & $96 \pm 45.3$ & $>128 \pm 0$ & + & 0.20 \\
\hline 5 & Vegetativo & A & $2.1 \pm 0.18$ & Mediana & $64 \pm 0.0$ & $>128 \pm 0$ & + & 0.28 \\
\hline 6 & Vegetativo & A & $1.6 \pm 0.10$ & Mediana & $16 \pm 0.0$ & $>128 \pm 0$ & + & 0.13 \\
\hline 7 & Vegetativo & A & $1.2 \pm 0.02$ & Mediana & $16 \pm 0.0$ & $>128 \pm 0$ & - & 0.47 \\
\hline 8 & Vegetativo & A & $6.0 \pm 0.07$ & Alta & $16 \pm 0.0$ & $>128 \pm 0$ & + & 0.21 \\
\hline 9 & Vegetativo & A & $5.2 \pm 0.05$ & Alta & $16 \pm 0.0$ & $>128 \pm 0$ & + & 0.2 \\
\hline 10 & Vegetativo & A & $5.0 \pm 0.1$ & Alta & $24 \pm 11.3$ & $>128 \pm 0$ & + & 0.17 \\
\hline 11 & Vegetativo & A & $6.1 \pm 0.03$ & Alta & $32 \pm 0.0$ & $>128 \pm 0$ & + & 0.23 \\
\hline 12 & Vegetativo & A & $5.8 \pm 0.03$ & Alta & $32 \pm 0.0$ & $>128 \pm 0$ & + & 0.00 \\
\hline 13 & Esporulado & A $\beta 2^{+v o s}$ & $0.6 \pm 0.01$ & Baja & $0 \pm 0.0$ & $0 \pm 0$ & - & 0.00 \\
\hline 14 & Esporulado & $\mathrm{A} \beta 2^{+v o s}$ & $-0.2 \pm 0.01$ & Baja & $0 \pm 0.0$ & $0 \pm 0$ & + & 0.00 \\
\hline 15 & Esporulado & $\mathrm{A} \beta 2^{+v o s}$ & $0.3 \pm 0.08$ & Baja & $0 \pm 0.0$ & $0 \pm 0$ & - & 0.00 \\
\hline 16 & Esporulado & A & $0.6 \pm 0.29$ & Baja & $2 \pm 0.0$ & $1 \pm 0$ & - & 0.21 \\
\hline 17 & Esporulado & A & $0.3 \pm 0.11$ & Baja & $3 \pm 1.4$ & $0 \pm 0$ & - & ND \\
\hline 18 & Esporulado & A & $0.4 \pm 0.05$ & Baja & $0 \pm 0.0$ & $0 \pm 0$ & - & 0.26 \\
\hline 19 & Esporulado & A & $0.4 \pm 0.05$ & Baja & $0 \pm 0.0$ & $2 \pm 0$ & - & 0.00 \\
\hline 20 & Esporulado & A & $0.4 \pm 0.08$ & Baja & $0 \pm 0.0$ & $2 \pm 0$ & - & 0.28 \\
\hline 21 & Esporulado & A & $0.5 \pm 0.32$ & Baja & $4 \pm 0.0$ & $2 \pm 0$ & - & 0.19 \\
\hline 22 & Esporulado & A & $0.5 \pm 0.04$ & Baja & $3 \pm 1.4$ & $2 \pm 0$ & - & 0.25 \\
\hline 23 & Esporulado & A & $0.7 \pm 0.03$ & Baja & $2.5 \pm 2.1$ & $2 \pm 0$ & - & 0.2 \\
\hline 24 & Esporulado & $\mathrm{A} \beta 2^{+v o s}$ & $1.0 \pm 0.06$ & Mediana & $0 \pm 0.0$ & $0 \pm 0$ & - & 0.00 \\
\hline
\end{tabular}

${ }^{1}$ Estado vegetativo considerado por características de crecimiento, morfología de la colonia y celular. Coloración Gram: bacilos gruesos y cortos de color azul intenso, sin presencia de esporas

${ }^{2}$ Genotipo A, poseen el gen plc [Cp-PLC] pero negativos a genes $c p b 2$ (toxina $\beta 2$ ) y cpe (CPE), Genotipo $A \beta 2^{+v o s}$, poseen genes p/c [Cp-PLC]) y $c p b 2$ (toxina $\beta 2$ ) pero carecen del gen $c p e$ (CPE)

${ }^{3} \mathrm{La}$ actividad lecitinasa expresada en unidades arbitrarias (u.a.) por mL. Calculada en forma triplicada para cada aislado, obteniéndose la media y la desviación estándar de las medias (SD)

${ }^{4}$ Capacidad de producción de Cp-PLC y clasificada por actividad lecitinasa: baja ( $<1$ u.a. $/ \mathrm{mL}$ ), mediana (1-3 u.a./mL) y alta (>3 u.a. $/ \mathrm{mL})$

${ }^{5}$ Actividad hemolítica siguiendo lo descrito por Titball et al. (1989), expresada en unidades arbitrarias hemolíticas (u.a.h.) por $\mathrm{mL}$ y calculada en forma duplicada (A y B) para cada aislado, obteniéndose la media y la desviación estándar de las medias (SD)

${ }^{6}$ Actividad perfringolisina, según la metodología descrita por Fisher et al. (2006), expresada en unidades arbitrarias hemolíticas (u.a.h.) por $\mathrm{mL}$ y calculada en forma duplicada (A y B) para cada aislado, obteniéndose la media y la desviación estándar de las medias (SD)

7 Actividad citotóxica evaluada en línea celular HEp-2. El despoblamiento celular (efecto citotóxico) fue considerado como una reacción positiva a citotoxicidad

${ }^{8}$ Capacidad de acumulación de fluido intestinal evaluada inoculando en asa intestinal ligada en conejo. Se evaluó la acumulación de fluido intestinal obtenida de la razón entre el volumen $(\mathrm{mL})$ y la longitud del asa intestinal ligada. 
Cuadro 2. Distribución de los aislados de C. perfringens de casos de enterotoxemia en alpacas, según la capacidad de producción de Cp-PLC, el estado bacteriano y el subtipo

\begin{tabular}{|c|c|c|c|c|c|c|c|}
\hline \multirow{2}{*}{$\begin{array}{c}\text { Capacidad } \\
\text { de producción } \\
\text { de Cp-PLC }\end{array}$} & \multicolumn{2}{|c|}{ Vegetativo $^{2}$} & \multirow{2}{*}{ Total } & \multicolumn{2}{|c|}{ Esporulado $^{3}$} & \multirow[b]{2}{*}{ Total } & \multirow{2}{*}{$\begin{array}{l}\text { Aislados } \\
\text { totales }\end{array}$} \\
\hline & $\beta 2^{-\operatorname{vos} 4}$ & $\beta 2^{+\operatorname{vos} 5}$ & & $\beta 2^{-v o s}$ & $\beta 2^{+v o s}$ & & \\
\hline Baja & $\begin{array}{c}20 \% \\
(2 / 10)\end{array}$ & $\begin{array}{l}100 \% \\
(2 / 2)\end{array}$ & $\begin{array}{l}33.3 \% \\
(4 / 12)\end{array}$ & $\begin{array}{c}100 \% \\
(8 / 8)\end{array}$ & $\begin{array}{l}75 \% \\
(3 / 4)\end{array}$ & $\begin{array}{l}91.6 \% \\
(11 / 12)\end{array}$ & $\begin{array}{c}62.5 \% \\
(15 / 24)\end{array}$ \\
\hline Mediana & $\begin{array}{c}30 \% \\
(3 / 10)\end{array}$ & - & $\begin{array}{c}25 \% \\
(3 / 12)\end{array}$ & - & $\begin{array}{l}25 \% \\
(1 / 4)\end{array}$ & $\begin{array}{l}8.4 \% \\
(1 / 12)\end{array}$ & $\begin{array}{l}16.6 \% \\
(4 / 24)\end{array}$ \\
\hline Alta & $\begin{array}{c}50 \% \\
(5 / 10)\end{array}$ & - & $\begin{array}{l}41.6 \% \\
(5 / 12)\end{array}$ & - & - & - & $\begin{array}{r}20.8 \% \\
(5 / 24)\end{array}$ \\
\hline \multicolumn{8}{|c|}{$\begin{array}{l}{ }^{1} \text { Capacidad de producción de Cp-PLC clasificada en base a su actividad lecitinasa: baja (<1 u.a./mL) } \\
\text { mediana (1-3 u.a./mL) y alta (>3 u.a./mL) } \\
2 \text { Estado vegetativo considerado por característica de crecimiento, morfología de la colonia y celular } \\
\text { Coloración Gram: bacilos gruesos y cortos de color azul intenso, sin presencia de esporas } \\
{ }^{3} \text { Estado esporulado considerado por característica de crecimiento, morfología de la colonia y celular } \\
\text { Coloración Gram: bacilos delgados y largos de color rosáceo, donde se pueden evidencia } \\
\text { fácilmente las endosporas } \\
{ }^{4} \text { Genotipo A } \beta 2^{-v o} \text {. Poseen gen plc [Cp-PLC]), que carecen de genes cpb2 (toxina } \beta 2 \text { ) y cpe (CPE) } \\
{ }_{5}^{5} \text { Genotipo A } \beta 2^{+v 0} \text {. Poseen genes plc [Cp-PLC]) y cpb2 (toxina } \beta 2 \text { ) pero carecen del gen cpe (CPE) }\end{array}$} \\
\hline
\end{tabular}

Cuadro 3. Actividades hemolíticas ${ }^{1}$ de los aislados de C. perfringens de casos de enterotoxemia en alpacas, según la capacidad de producción de Cp-PLC, estado bacteriano y subtipo

\begin{tabular}{|c|c|c|c|c|c|c|c|}
\hline \multirow{2}{*}{$\begin{array}{l}\text { Capacidad } \\
\text { de } \\
\text { producción } \\
\text { de Cp-PLC }\end{array}$} & \multicolumn{3}{|c|}{ Vegetativo $^{3}$} & \multicolumn{3}{|c|}{ Esporulado ${ }^{4}$} & \multirow{2}{*}{ Total } \\
\hline & $\beta 2^{-\operatorname{vos} 5}$ & $\beta 2^{+\operatorname{vos} 6}$ & Total & $\beta 2^{-10 s}$ & $\beta 2^{+v o s}$ & Total & \\
\hline Baja & $49.5 \pm 59.7^{\mathrm{a}}$ & 0 & 24.8 & $8 \pm 1.7$ & 0 & $31 \pm 1.7$ & $7.6 \pm 25.5^{\mathrm{a}}$ \\
\hline Mediana & $32.0 \pm 24.8^{\mathrm{a}}$ & - & $32.0 \pm 24.8^{\mathrm{a}}$ & - & 0 & 0 & $24.0 \pm 25.7^{\mathrm{a}}$ \\
\hline Alta & $24.0 \pm 8.4^{\mathrm{a}}$ & - & $1.0=$ & - & - & - & 24.0 \\
\hline Total & $31.5 \pm 29.3$ & 0 & $26.3 \pm 29.1$ & $1.8 \pm 1.7$ & 0 & $1.2 \pm 1.64$ & $13.7 \pm 24.0$ \\
\hline \multicolumn{8}{|c|}{$\begin{array}{l}{ }^{a} \text { Sin diferencia estadística significativa a la prueba de ANOVA simple aleatoria al } 95 \% \text { de confianza } \\
{ }^{1} \text { Actividad hemolítica según la metodología descrita por Titball et al. (1989), expresada en unidades } \\
\text { arbitranias hemolíticas (u.a.h.) por mL y calculada por duplicado para cada aislado, obteniéndose la } \\
\text { media y la desviación estándar de las medias (SD) } \\
{ }^{2} \text { Capacidad de producción de Cp-PLC clasificada por su actividad lecitinasa: baja (<1 u.a./mL), } \\
\text { mediana (1-3 u.a./mL) y alta ( }>3 \text { u.a./mL) } \\
{ }^{3} \text { Estado vegetativo, considerado por característica de crecimiento, morfología de colonia y celular. } \\
\text { Coloración Gram: bacilos gruesos y cortos de color azul intenso, sin presencia de esporas } \\
{ }^{4} \text { Estado esporulado, considerado por característica de crecimiento, morfología de colonia y celular. } \\
\text { Coloración Gram: bacilos delgados y largos de color rosáceo y presencia de endosporas } \\
{ }_{5} \text { Genotipo A } \beta 2^{-v 0} \text {. Posee gen plc [Cp-PLC]) pero carecen de gen cpb2 (toxina } \beta 2 \text { ) y gen } c p e \text { (CPE) } \\
{ }^{6} \text { Genotipo A } \beta 2^{+v 0} \text {. Poseen genes plc [Cp-PLC]) y cpb2 (toxina } \beta 2 \text { ) pero carecen del gen cpe (CPE) }\end{array}$} \\
\hline
\end{tabular}


Cuadro 4. Actividades perfringolisinas ${ }^{1}$ de los aislados de C. perfringens de casos de enterotoxemia en alpacas, según capacidad de producción de Cp-PLC, estado bacteriano y subtipo

\begin{tabular}{|c|c|c|c|c|c|c|c|}
\hline \multirow{2}{*}{$\begin{array}{l}\text { Capacidad } \\
\text { de } \\
\text { producción } \\
\text { de Cp-PLC }\end{array}$} & \multicolumn{3}{|c|}{ Vegetativo $^{3}$} & \multicolumn{3}{|c|}{ Esporulado $^{4}$} & \multirow{2}{*}{ Total } \\
\hline & $\beta 2^{-v o s 5}$ & $\beta 2^{+\operatorname{vos} 6}$ & Total & $\beta 2^{-v o s}$ & $\beta 2^{+v o s}$ & Total & \\
\hline Baja & $65 \pm 72^{\mathrm{a}}$ & 0 & $33 \pm 59^{\mathrm{a}}$ & $1.4 \pm 0.9$ & 0 & $1 \pm 1$ & $9 \pm 32^{\mathrm{a}}$ \\
\hline Mediana & $128 \pm 0^{\mathrm{a}}$ & - & $128 \pm 0^{\mathrm{b}}$ & - & 0 & 0 & $96 \pm 59^{b}$ \\
\hline Alta & $128 \pm 0^{\mathrm{a}}$ & - & $128 \pm 0^{\mathrm{b}}$ & - & - & 0 & $128 \pm 0^{\mathrm{b}}$ \\
\hline Total & $115 \pm 38$ & 0 & $96 \pm 56$ & $1.4 \pm 0.9$ & 0 & $0.9 \pm 1$ & $48 \pm 62$ \\
\hline \multicolumn{8}{|c|}{$\begin{array}{l}\text { a,b } \text { Diferencia estadística significativa a la prueba de ANOVA simple aleatorio al } 95 \% \text { de confianza } \\
{ }^{1} \text { Actividad perfringolisina determinada según Fisher et al. (2006), expresada en unidades arbitrarias } \\
\text { hemolíticas (u.a.h.) por } \mathrm{mL} \text { y calculada por duplicado, obteniéndose la media y la desviación } \\
\text { estándar de las medias (SD). Valores obtenidos asumiendo el valor }>128 \text { u.a.h/mL como } 128 \\
\text { u.a.h./mL } \\
{ }^{2} \text { Capacidad de producción de Cp-PLC clasificada por actividad lecitinasa: baja ( }<1 \text { u.a./mL), } \\
\text { mediana (1-3 u.a./mL) y alta ( }>3 \text { u.a./mL) } \\
{ }^{3} \text { Estado vegetativo, considerado por características de crecimiento, morfología de colonia y celular. } \\
\text { Coloración Gram: bacilos gruesos y cortos de color azul intenso, sin presencia de esporas } \\
{ }^{4} \text { Estado esporulado, considerado por características de crecimiento, morfología de colonia y celular. } \\
\text { Coloración Gram: bacilos delgados y largos de color rosáceo, con presencia de endosporas } \\
{ }^{5} \text { Genotipo A } \beta 2^{-v o} \text {. Posee el gen plc [Cp-PLC]) pero carecen de los genes cpe (CPE) y cpb2 (toxina } \\
\beta 2 \text { ) } \\
{ }^{6} \text { Genotipo A } \beta 2^{+v o} \text {. Poseen genes plc [Cp-PLC]) y cpb2 (toxina } \beta 2 \text { ) pero carecen del gen cpe (CPE) }\end{array}$} \\
\hline
\end{tabular}

la escasa producción de Cp-PLC o la ausencia de hemolisinas tipo Cp-PLC de manera similar a reportes en aislados $C$. perfringens genotipo C $c p b 2^{+\mathrm{vo}}$ (Fisher et al., 2006).

La producción de PFO en los aislados vegetativos $(>96 \pm 56$ u.a.h. $/ \mathrm{mL})$ fue superior a los aislados esporulados $(0.9 \pm 1$ u.a.h./ $\mathrm{mL}$ ) (Cuadro 4). La actividad perfringolisina fue directamente proporcional a la capacidad de producción de Cp-PLC, pues los mayores valores de actividad perfringolisina fueron detectados en aislados vegetativos de alta y mediana producción $(>128 \pm 0$ u.a.h./mL) comparados con los de baja de producción $(33 \pm 59$ u.a.h./mL) (Cuadro 4). Estos resultados eran esperados, pues la perfringolisina, de manera similar a la Cp-PLC, se sintetiza durante la fase de crecimiento exponencial y es regulada por el mismo sistema (VirR/VirS) que la Cp-PLC (Rood, 1998). Interesan- temente, ninguno de los aislados subtipo $\hat{a} 2^{+v o}$ (vegetativos y esporulados) presentaron actividad perfringolisina, sugiriendo un posible efecto pleitrópico negativo del gen $c p b 2 \mathrm{u}$ otros genes relacionados en la expresión de los genes cpa (Cp-PLC) y pfoA (codificante de PFO).

La actividad citotóxica de los aislados observada en cultivos celulares HEp-2 fue proporcional a los niveles de producción de Cp-PLC, evidenciando claramente actividades citotóxicas de la Cp-PLC pero dependiente de concentraciones. Todos los aislados en estados vegetativos de mayores producciones fueron citotóxicas, mientras que el $66.7 \%$ de mediana producción y el $50 \%$ de bajas productoras mostraron efectos citotóxicos (Cuadro 5). Por otro lado, los aislados en estado de esporulación mostraron una escasa o nula actividad citotóxica $(0.1 \%)$. Asimismo, 2 de 4 aislados (vegetativos) de 
Cuadro 5. Actividad citotóxica ${ }^{1}(\%)$ de los aislados de C. perfringens de casos de enterotoxemia en alpacas, según su capacidad de producción de Cp-PLC, estado bacteriano y subtipo

\begin{tabular}{|c|c|c|c|c|c|c|c|}
\hline \multirow{2}{*}{$\begin{array}{l}\text { Capacidad de } \\
\text { producción de } \\
\text { Cp-PLC }\end{array}$} & \multicolumn{3}{|c|}{ Vegetativo $^{3}$} & \multicolumn{3}{|c|}{ Esporulado $^{4}$} & \multirow{2}{*}{ Total } \\
\hline & $\beta 2^{-\operatorname{vos} 5}$ & $\beta 2^{+\operatorname{vos} 6}$ & Total & $\beta 2^{-v o s}$ & $\beta 2^{+v o s}$ & Total & \\
\hline Baja & $\begin{array}{l}50 \% \\
(1 / 2)\end{array}$ & $\begin{array}{l}50 \% \\
(1 / 2)\end{array}$ & $(2 / 4)$ & $\begin{array}{c}0 \% \\
(0 / 8)\end{array}$ & $\begin{array}{c}33.3 \% \\
(1 / 3)\end{array}$ & $\begin{array}{l}0.09 \% \\
(1 / 11)\end{array}$ & $\begin{array}{l}0.2 \% \\
(3 / 15)\end{array}$ \\
\hline Mediana & $\begin{array}{c}66.7 \% \\
(2 / 3)\end{array}$ & - & $\begin{array}{c}66.7 \% \\
(2 / 3)\end{array}$ & - & $\begin{array}{c}0 \% \\
(0 / 1)\end{array}$ & $\begin{array}{c}0 \% \\
(0 / 1)\end{array}$ & $\begin{array}{l}50 \% \\
(2 / 4)\end{array}$ \\
\hline Alta & $\begin{array}{c}100 \% \\
(5 / 5)\end{array}$ & & $\begin{array}{c}100 \% \\
(5 / 5)\end{array}$ & & - & - & $\begin{array}{c}100 \% \\
(5 / 5)\end{array}$ \\
\hline Total & $\begin{array}{c}80 \% \\
(8 / 10)\end{array}$ & $\begin{array}{l}50 \% \\
(1 / 2)\end{array}$ & $\begin{array}{c}75 \% \\
(9 / 12)\end{array}$ & $\begin{array}{c}0 \% \\
(0 / 8)\end{array}$ & $\begin{array}{l}25 \% \\
(1 / 4)\end{array}$ & $\begin{array}{l}0.08 \% \\
(1 / 12)\end{array}$ & $\begin{array}{c}41.7 \% \\
(10 / 24)\end{array}$ \\
\hline \multicolumn{8}{|c|}{$\begin{array}{l}{ }^{1} \text { Actividad citotóxica en línea celular HEp-2. El despoblamiento celular (efecto citopático) fue } \\
\text { considerado como una reacción positiva a citotoxicidad } \\
2 \text { Capacidad de producción de Cp-PLC clasificada por actividad lecitinasa: baja (<1 u.a./mL), mediana } \\
\text { (1-3 u.a./mL) y alta ( }>3 \text { u.a./mL) } \\
3 \text { Estado vegetativo, considerando características de crecimiento, morfología de colonia y celular. } \\
\text { Coloración Gram: bacilos gruesos y cortos de color azul intenso, sin presencia de esporas } \\
{ }^{4} \text { Estado esporulado, considerando características de crecimiento, morfología de colonia y celular. } \\
\text { Coloración Gram: bacilos delgados y largos de color rosáceo y presencia de endosporas } \\
{ }^{5} \text { Genotipo A } \beta 2^{-v 0} \text {. Poseen gen plc [Cp-PLC]), pero carecen de genes cpb2 (toxina } \beta 2 \text { y cpe (CPE) } \\
{ }^{6} \text { Genotipo A } \beta 2^{+v 0} \text {. Poseen genes plc [Cp-PLC]) y cpb2 (toxina } \beta 2 \text { ), pero carecen del gen cpe (CPE) }\end{array}$} \\
\hline
\end{tabular}

baja producción de Cp-PLC y 2 de 5 subtipos $\hat{a} 2^{+\mathrm{vo}}$ mostraron similares actividades citotóxicas (Cuadro 5), evidenciando la posibilidad de existencia de otras citotoxinas distintas a la Cp-PLC o efectos directos asociados a la toxina $\hat{a} 2$.

Los aislados de mediana producción de Cp-CLC produjeron las mayores acumulaciones de fluido intestinal en el sitio de inoculación (0.29 \pm 0.17$)$, seguidas muy cercanamente por los aislados de alta producción $(0.16 \pm 0.09 \mathrm{~mL} / \mathrm{cm})$ y por los de baja producción $(0.10 \pm 0.12 \mathrm{~mL} / \mathrm{cm})(\mathrm{Cua}-$ dro 6). Estos datos sugieren cierta capacidades de acumular fluido intestinal por aislados de baja capacidad de producción, independiente de las cantidades de fosfolipasas C. La relativa pobre producción de los aislados altamente productores de fosfolipasas eviden- cia la poca capacidad de esta toxina para inducir acumulación de fluido intestinal, de manera similar a lo observado en una cepa de $C$. perfringens tipo A $c p e^{- \text {-vo }} c p b 2^{\text {-vo }}$ inoculado experimentalmente en asas intestinales de cordero tratado con sueros anti-CpPLC sin impedir formación de fluido (Hauschild et al., 1968). Por otro lado, la habilidad de acumular fluido por los aislados mediamente productores de Cp-PLC sugiere la presencia de toxinas similares a enterotoxinas con capacidades de estimular la secreción de fluido sin causar alteraciones tisulares (Sear y Kaper, 1996).

Los aislados en estados esporulados, negativos al gen cpe, fueron igualmente capaces de desencadenar acumulación de fluido intestinal ( $0.14 \pm 0.12 \mathrm{~mL} / \mathrm{cm}$, Cuadro 6), corroborando lo observado experimentalmen- 
Cuadro 6. Promedios de acumulación de fluido intestinal ${ }^{1}$ (relación volumen/longitud de AIL) de los aislados de $C$. perfringens de casos de enterotoxemia en alpacas, según su capacidad de producción de Cp-PLC, estado bacteriano y subtipo

\begin{tabular}{lccccccc}
\hline $\begin{array}{l}\text { Capacidad } \\
\text { de }\end{array}$ & \multicolumn{3}{c}{ Vegetativo $^{3}$} & \multicolumn{3}{c}{ Esporulado $^{4}$} & Total \\
\cline { 2 - 7 } $\begin{array}{l}\text { producción } \\
\text { de Cp-PLC }\end{array}$ & $\beta 2^{\text {-vos } 5}$ & $\beta 2^{+v o s} 6$ & Total & $\beta 2^{\text {-vos }}$ & $\beta 2^{+v o s}$ & Total & \\
\hline Baja & $0.21 \pm 0.01^{\mathrm{a}}$ & 0 & $0.10 \pm 0.12^{\mathrm{a}}$ & $0.20 \pm 0.09$ & 0 & $0.14 \pm 0.12$ & $0.13 \pm 0.12^{\mathrm{a}}$ \\
Mediana & $0.29 \pm 0.17^{\mathrm{a}}$ & - & $0.29 \pm 0.17^{\mathrm{a}}$ & - & 0 & 0 & $0.22 \pm 0.20^{\mathrm{a}}$ \\
Alta & $0.16 \pm 0.09^{\mathrm{a}}$ & - & $0.16 \pm 0.09^{\mathrm{a}}$ & - & - & - & $0.16 \pm 0.09^{\mathrm{a}}$ \\
\hline Total & $0.21 \pm 0.12$ & 0 & $0.18 \pm 0.13$ & $0.20 \pm 0.09$ & 0 & $0.15 \pm 0.13$ & $0.12 \pm 0.13$ \\
\hline
\end{tabular}

a: Sin diferencia estadística significativa mediante el uso de ANOVA simple aleatorio al 95\% de confianza

${ }^{1}$ La acumulación de fluido intestinal fue determinada según la metodología descrita en el presente estudio usando la prueba de asa intestinal ligada en conejos. Valores expresados corresponden a las medias de la razón entre el volumen $(\mathrm{mL})$ de fluido intestinal en el asa intestinal ligada y la longitud (cm) del asa intestinal ligada, con la respectiva desviación estándar de las medias (SD)

2 Capacidad de producción de Cp-PLC clasificada por su actividad lecitinasa: baja ( $<1$ u.a. $/ \mathrm{mL}$ ), mediana (1-3 u.a./mL) y alta (>3 u.a. $/ \mathrm{mL}$ )

3 Estado vegetativo, basado por características de crecimiento, morfología de colonia y celular. Coloración Gram: bacilos gruesos y cortos de color azul intenso, sin presencia de esporas

${ }^{4}$ Estado esporulado, por características de crecimiento, morfología de colonia y celular. Coloración Gram: bacilos delgados y largos de color rosáceo y presencia de endosporas

${ }^{5}$ Genotipo $A \beta 2^{-v o}$. Poseen gen p/c [Cp-PLC]), pero carecen de genes cpb2 (toxina $\beta 2$ ) y cpe (CPE)

${ }^{6}$ Genotipo $A \beta 2^{+v o}$. Poseen genes plc [Cp-PLC]) y cpb2 (toxina $\beta 2$ ), pero carecen de cpe (CPE)

te en alpacas después de inocular en asas intestinales con células esporuladas de $C$. perfringens y que fue atribuido a la enterotoxina (Ramírez, 1987). Los resultados del presente estudio, sin embargo, evidencian presencia de otras enterotoxinas distintas a la CPE en estos aislados que estarían siendo sintetizadas, tanto en su estado vegetativo como esporulado.

Todos los aislados subtipo $\hat{a} 2^{+v o}$ fueron incapaces de producir acumulación de fluido (Cuadro 6), y menos aún, inducir lesiones hemorrágicas, contrastando lo descrito sobre la toxina $\hat{2} 2$ (Schotte et al., 2004). Similares incapacidades han sido observadas en inoculaciones con cultivos de $C$. perfringens tipo A $c p b 2^{+v o}$ en yeyuno de vacunos (Ewoldt y Anderson, 2005). Esta incapacidad enterotóxica tal vez responda a que la toxina â2 no habría sido sintetizada en los aislados, pues la expresión de gen $c p b 2$ es irregular y dependiente del medio de cultivo empleado (medios específicos suplementados con antibióticos) (Vilei et al., 2005) o del tipo de alelo $c p b 2$ involucrado, que casi siempre se expresa en su forma consensus o pocas veces en lo atípico (Lebrun et al., 2007).

Ningún aislado fue capaz de provocar lesiones degenerativas, necróticas o hemorrágicas en la mucosa, submucosa o serosa intestinal similares a los descritos como lesiones típicas de enterotoxemia. Estos resultados tienden a descartar el rol patogénico de la Cp-PLC en la producción de lesiones entéricas por $C$. perfringens tipo A de manera similar a lo encontrado en desafíos ex- 
perimentales con sobrenadante de cultivos de C. perfringens tipo A $c p e^{-\mathrm{vo}} c p b 2^{-\mathrm{vo}}$ en asas intestinales de terneros (Manteca et al., 2002), corderos (Hauschild et al., 1968). La incapacidad de inducir lesiones enterotóxicas tal vez sea consecuencia de inocular especies bacterianas de alpacas a conejos, pues existen reportes sobre la incapacidad de inducir lesiones en asas intestinales de pollos utilizando cepas de alta capacidad de producción de Cp-PLC de $C$. perfringens aisladas de terneros, aunque mostraron capacidad enterotóxica cuando se utilizaron cepas de $C$. perfringens recuperadas de pollos enfermos, independiente de su capacidad de producción de Cp-PLC (Timbermont et al., 2009). Sin embargo, inoculaciones experimentales en asas intestinales de alpacas neonatas con sobrenadantes de $C$. perfringens de alta y mediana producción de Cp-PLC tampoco reprodujeron lesiones enterotóxicas tisulares (Á. Veliz, comunicación personal).

Los resultados señalan que la Cp-PLC presente en sobrenadantes crudos no sería el principal factor de virulencia responsable de las lesiones entéricas características, reportadas en los casos de enterotoxemia de alpacas. Esta toxina tiene capacidad de producir lesiones citotóxicas y actividades hemolíticas que podrían explicar posibles acciones patológicas al alcanzar nivel sistémico, y producir desórdenes multi-orgánicos que han sido descritos en la enterotoxemia de alpacas. En este esquema, el desencadenamiento de la enterotoxemia dependería de acciones patogénicas sinérgicas o de co-infecciones con infecciones primarias de una diversidad de patógenos entéricos, entre los cuales se encuentran las infecciones coccidiales descritas por Rosadio et al. (2010) o por secreciones de shigatoxinas de E. coli, las cuales han sido demostradas en alpacas con cuadros de enterotoxemia (Luna, 2009).

Asimismo, los sobrenadantes bacterianos crudos contienen otras toxinas tipo hemolisinas, citotoxinas y toxinas entéricas, diferentes a Cp-PLC, PFO, y CPE, que fueron similares o más agresivas que la Cp-PLC bajo las condiciones analizadas. La variedad de exoproteínas presentes en estos sobrenadantes, de bacterias en estado vegetativos y esporulados, han sido detectadas mediante la técnica SDS-PAGE (Electroforesis en Gel de Poliacrilamida con Sodio Dodecil Sulfato), teniendo un patrón electroforético constituido aproximadamente por 16 productos, con pesos moleculares de $27 \mathrm{KDa}-97 \mathrm{KDa}$, bacterianos, mostrando in situ presencia de una gama de toxinas que necesitan ser analizadas para elucidar posible participación en el complejo entero-toxémico (Pérez, 2010). Similares observaciones han permitido conducir al descubrimiento de importantes factores de virulencias en otras enteropatías clostridiales, tales como la enteritis necrótica en pollos, que permitieron identificar y caracterizar la toxina NetB (Keyburn et al., 2008).

\section{Conclusiones}

- Se describe y reporta, por primera vez, la presencia de tres subtipos de $C$. perfringens asociados a enterotoxemia en alpacas con distintos grados de producción de la Cp-PLC: baja, mediana y alta. Los sobrenadantes nativos (crudos) muestran distintas capacidades hemolíticas y todas fueron citotóxicas para las células Hep2.

- Se encontraron distintas capacidades para acumular fluido en intestinos ligados de conejo sin guardar relación con la producción de la Cp-PLC, pero todas fueron incapaces de inducir lesiones patológicas similares a las descritas en casos de enterotoxemia.

- La Cp-PLC no sería un factor de virulencia esencial en la generación de lesiones entéricas.

- Los sobrenadantes crudos de estos aislados evidencian presencia de otras toxinas tipo hemolisinas, citotoxinas y enterotoxinas, distintas a Cp-PLC, CPE y toxina $\hat{a} 2$. 
- No hubo diferencias estadísticas entre la actividad hemolítica y acumulación de fluidos entre cepas de baja, media y alta producción de Cp-PLC, pero se detectó diferencia estadística entre actividad de perfringolisina entre las cepas de baja y las cepas de mediana y alta producción de Cp-PLC.

\section{LiTERATURA CITADA}

1. Ameghino E, DeMartini J. 1991. Mortalidad de crías de alpacas. En: Boletín de Divulgación del Instituto Veterinario de Investigaciones Tropicales y de Altura (IVITA). Lima: UNMSM. p 71-80.

2. Ba-Thein W, Lyristis M, Ohtani K, Inisbet IT, Hayashi H, Rood JL, Shimizu T. 1996. The virR/virS locus regulates the transcription of genes encoding extracellular toxin production in Clostridium perfringens. J Bacteriol 178: 2514-2520.

3. Bullifent HL, Moir A, Awad MM, Scott PT, Rood JI, Titball RW. 1996. The level of expression of á-toxin by different strains of Clostridium perfringens is dependent on differences in promote structure and genetic background. Anaerobe 2: 365-371.

4. Campos D de FS, Baccaro MR, Moreno AM, Ferreira AJP, Doto DS, Murakami DF, Shinya LT. 2004. Clostridium perfringens tipo A portadores do gene $\mathrm{cpb} 2$ associados à lesões em íleo de coelhos. Arq Inst Biol São Paulo 71: 287-292.

5. Duncan CL, Sugiyama H, Strong DH. 1968. Rabbit ileal loop response to strains of Clostridium perfringens. $\mathrm{J}$ Bacteriol 95: 1560-1566.

6. Embury-Hyatt CK, Wobeser G Simko E, Woodbury MR. 2005. Investigation of a syndrome of sudden death, splenomegaly, and small intestinal hemorrhage in farmed deer. Can Vet J 46: 702-708.

7. Ewoldt JM, Anderson DE. 2005. Determination of the effect of single abomasal or jejuna inoculation of Clostridium perfringens type A in dairy cows. Can Vet J 46: 821-824.

8. Fiore AE, Michalski JM, Russell RG, Sears CL, Kaper JB. 1997. Cloning, characterization, and chromosomal mapping of a phospholipase (lecitinase) produced by Vibrio cholera. Infect Immun 65:3112-3117.

9. Fisher DJ, Fernandez-Miyakawa ME, Sayeed S, Poon R, Adams V, Rood JI, Uzal FA, McClane BA. 2006. Dissecting the contributions of Clostridium perfringens type $\mathrm{C}$ toxins to lethality in the mouse intravenous injection model. Infect Immun 74: 52005210.

10. Flores-Díaz M, Alape-Girón A. 2003. Role of Clostridium perfringens phospholipase $\mathrm{C}$ in the pathogenesis of gas gangrene. Toxicon 42: 979-986.

11. Gholamiandekhordi AR, Ducatelle $R$, Heyndrickx M, Haesebrouck F, Immerseel FV. 2006. Molecular and phenotypical characterization of Clostridium perfringens isolates from poultry flocks with different disease status. Vet Microbiol 113: 143-152.

12. Gkiourtzidis K, Frey J, BourtziHatzopoulou E, Iliadis N, Sarris K. 2001. PCR detection and prevalence of á-, â-, â2-, å-, é and enterotoxin genes in Clostridium perfringens isolated from lambs with clostridial dysentery. Vet Microbiol 82: 39-43.

13. Hauschild AHW, Niilo L, Dorward WJ. 1968. Clostridium perfringens type A infection of ligated loops in lambs. Appl Microbiol 16: 1235-1239.

14. Kalender H, Ertas HB, Ceninkaya B, Muz A, Aislan N, Kilic A. 2005. Typing of isolates of Clostridium perfringens from healthy and diseased sheep by multiplex PCR. Vet Med-Czech 50:439-442.

15. Kanakaraj R, Harris DL, Songer JG, Bosworth B. 1998. Multiplex PCR assay for detection of Clostridium perfringens in feces and intestinal contents of pigs and swine feed. Vet Microbiol 63: 29-38. 
16. Katayama S, Matsushita $O$, Minami J, Mizobuchi S, Okabe A. 1993. Comparison of the alpha-toxin genes of Clostridium perfringens type $\mathrm{A}$ and $\mathrm{C}$ strains: evidence for extragenic regulation of transcription. Infect Immun 61: 457-463.

17. Keyburn AL, Boyce JD, Vaz P, Bannam TL, Ford ME, Parker D, Di Rubbo A, et al. 2008. NetB, a new toxin that is associated with avian necrotic enteritis caused by Clostridium perfringens. PLoS Pathog 4: 1-11.

18. Lebrun M, Filée P, Mousset B, Desmecht D, Galleni M, Mainil JG, Linden A. 2007. The expression of Clostridium perfringens consensus beta 2 toxin is associated with bovine enterotoxaemia syndrome. Vet Microbiol 120: 151-157.

19. Luna E. 2009. Genotipificación de cepas de Escherichia coli aislados de crías de alpacas con diarrea. Tesis de Médico Veterinario. Lima: Univ Nac Mayor de San Marcos. 73 p.

20. Manteca C, Daube G, Jauniaux T, Linden A, Pirson V, Detilleux J, Ginter $A$, et al. 2002. A role for the Clostridium perfringens â2 toxin in bovine enterotoxaemia? Vet Microbiol 86: 191-202.

21. Moro M. 1987. Enfermedades infecciosas de las alpacas. Diarrea bacilar o enterotoxemia de las crías de las alpacas. Rev Camélidos Sudamericanos 4: 8-13.

22. Nagahama M, Michiue K, Sakurai J. 1996. Membrane-damaging action of Clostridium perfringens alpha-toxin on phospholipid liposomes. Biochim Biophys Acta 1280: 120-126.

23. Pérez D. 2006. Genotipificación y subtipificación de Clostridium perfringens aisladas de crías de alpacas muertas por enterotoxemia. Tesis de Médico Veterinario. Lima: Univ Nac Mayor de San Marcos. 91 p.

24. Pérez D. 2010. Caracterización toxigénica de la fosfolipasa $\mathrm{C}$ del Clostridium perfringens (Cp-PLC) y su relación con aislados de $C$. perfringens de casos de enterotoxemia en alpacas. Tesis de Magíster. Lima: Univ Nac Mayor de San Marcos. 151 p.

25. Ramírez A. 1991. Enfermedades infecciosas. En: Fernández S (eds). Avances y perspectivas del conocimiento de los camélidos sudamericanos. Santiago de Chile: FAO. p 265-289.

26. Ramírez A. 1987. Alpaca Clostridium perfringens type A enterotoxemia: Purification and assays of the enterotoxin. PhD Thesis. Colorado: Colorado State University. $130 \mathrm{p}$.

27. Rood J. 1998. Virulence genes of Clostridium perfringens. Annu Rev Microbiol 52: 333-360.

28. Rood J, Cole S. 1991. Molecular genetics and pathogenesis of Clostridium perfringens. Microbiol Rev 55: 621-648.

29. Rosadio R, Londoñe P, Pérez D, Castillo $H$, Véliz A, Llanco L, Yaya $K$, Maturrano L. 2010. Eimeria macusaniensis associated lesions in neonate alpacas dying from enterotoxemia. Vet Parasitol 168: 116-120.

30. Schotte U, Truyen U, Neubauer $\boldsymbol{H}$. 2004. Significance of â2-toxigenic Clostridium perfringens infections in animals and their predisposing factors. J Vet Med 51: 423-426.

31. Sear CL, Kaper JB. 1996. Enteric bacterial toxins: mechanisms of action and linkage to intestinal secretion. Microbiol Rev 60: 167-215.

32. Sheedy SA, Ingham AB, Rood JI, Moore RJ. 2004. Highly conserved alpha-toxin sequences of avian isolates of Clostridium perfringens. J Clin Microbiol 42: 1345-1347.

33. Timbermont L, Lanckriet A, Gholamiandehkordi AR, Pasmans F, Martel A, Haesebrouck F, Ducatelle $\boldsymbol{R}$, Van Immerseel F. 2009. Origin of Clostridium perfringens isolates determines the ability to induce necrotic enteritis in broilers. Comp Immunol Microbiol Infect Dis 32: 503-512. 
34. Titball RW, Hunter SEC, Martin KL, Morris BC, Shuttleworth AD, Rubidge T, Anderson DW, Kelly DC. 1989. Molecular cloning and nucleotide sequence of the alpha-toxin (phospholipase C) of Clostridium perfringens. Infect Immum 57: 367-376.

35. Titball RW. 2005. Gas gangrene: an open and closed case. Microbiology 151: 2821-2828.

36. Titball RW, Naylor CE, Basak AK. 1999. The Clostridium perfringens átoxin. Anaerobe 5: 51-64.
37. Tsutsui K, Minami J, Matsushita O, Katayama S, Taniguchi Y, Nakamura S, Nishioka M, Okabe A. 1995. Phylogenetic analysis of phospholipase $\mathrm{C}$ genes from Clostridium perfringens types A to E and Clostridium novyi. J Bacteriol 177: 7164-7170.

38. Vilei EM, Schlatter Y, Perreten V, Strau R, Popoff MR, Gibert M, Gönem A, Frey J. 2005. Antibiotic-induced expression of a cryptic cpb2 gene in equine â2-toxigenic Clostridium perfringens. Mol Microbiol 57: 1570-1581. 